\title{
Sound pressure attenuation provided by a 3D rigid acoustic barrier on a building façade: the influence of its longitudinal shape
}

\author{
A. Tadeu ${ }^{1}$, J. António $^{1} \&$ I. Castro ${ }^{2}$ \\ ${ }^{I}$ Department of Civil Engineering, University of Coimbra, Portugal \\ ${ }^{2}$ Research and Technological Development in Construction \\ Sciences - ITeCons, Portugal
}

\begin{abstract}
This paper models the propagation of sound in the vicinity of $3 \mathrm{D}$ acoustic barriers placed parallel to a building façade to mitigate the noise generated by point pressure sources. The barriers are assumed to be very thin rigid elements. The problem is solved by developing and implementing a 3D boundary element method formulation based on the normal derivative integral equation (TBEM). The TBEM is formulated in the frequency domain and the resulting hypersingular terms are computed analytically.

After verifying the model against 2.5D BEM solutions, several numerical applications are described to illustrate the practical usefulness of the proposed approaches. Different longitudinal barrier geometries are simulated to evaluate the influence of this characteristic on the sound pressure level attenuation attained at the building façade.
\end{abstract}

Keywords: acoustic wave propagation, $3 D$ thin barriers, normal derivative integral equation, analytical integration of hypersingular integrals.

\section{Introduction}

Different numerical methods have been developed to solve acoustic problems in either the time domain or the frequency domain. The works by Marburg and Nolte [1], Cheng and Cheng [2] and Pluymers et al. [3] and the reference book by Jensen et al. [4] give a good general overview of the developments in this field. 
Of the various numerical methods the boundary element method is perhaps the best one to model acoustic barriers since it can easily account for infinite or semi-infinite domains and automatically satisfy the far-field radiation conditions. As it is based on fundamental solutions established for infinite spaces, the method can be successfully used to model scenarios of outdoor sound propagation or underwater propagation in which the propagation media may be described as unbounded domains (Grilli et al. [5], Santiago and Wrobel [6], Pereira et al. [7]).

However, the BEM cannot be directly applied to problems which involve very thin objects such as acoustic screens because the establishment of the boundary integral equations on both sides of the object leads to a singular equation system. Special solution strategies can be adopted that avoid this problem. The so-called dual BEM formulation is one such strategy (as reported in Portela et al. [8], Chen and Hong [9] and Krishnasamy et al. [10]): in it, both the classic boundary integral equation and its first spatial derivative along the orthogonal direction to the boundary are used to obtain a non-singular system of equations. If the thickness of the obstacle is null a very effective Traction-BEM (TBEM) formulation can also be used (see e.g. [11]) which enables the obstacle to be described by means of a single line of boundary elements. The dual BEM and the TBEM techniques have been successfully used to study acoustic wave scattering in the presence of thin barriers (Lacerda et al. [12, 13], António et al. [14]).

Most of the works mentioned above simulated two-dimensional barriers, assuming either the incidence of pressure waves generated by two (2D formulation) or three-dimensional pressure sources (2.5D formulation). Despite the computational restrictions of 3D BEM formulations when it comes to solving large acoustic problems, they are suitable for solving small-scale problems and give a good approximation of the $3 \mathrm{D}$ sound field.

In this paper the authors address the use of a frequency-domain BEM formulation based on the normal derivative integral equation to model sound propagation in $3 \mathrm{D}$ configurations incorporating very thin screens. One of the most significant difficulties posed by this formulation is the integration of hypersingular integrals that are here performed analytically.

In the sections that follow, the three-dimensional problem is presented and the three-dimensional normal derivative integral equation described. The strategy devised to solve the hypersingular integrals analytically is then explained. Finally, a method to obtain time-domain responses from frequency-domain calculations is described and a number of numerical applications are presented. These applications mainly focus on the effect of the longitudinal geometry of the acoustic barriers, used to mitigate the noise generated by 3D point sources, on a building façade, and the sound pressure level attenuation is calculated.

\section{3D normal derivative integral equation (3D TBEM)}

This section first describes how the 3D TBEM is formulated to obtain the scattered acoustic pressure wave field, i.e. the pressure in the host medium 
generated by the incident three-dimensional pressure waves illuminating a 3D barrier, in an unbounded medium. The simulation of the sound propagation when the barrier is in a corner space that represents a floor and a building façade is presented afterwards.

\subsection{Unbounded medium}

Consider a three-dimensional barrier, bounded by a surface, $S$, in a spatially uniform acoustic medium of density $\rho$, where the pressure waves propagate at velocity $c$ (see Figure 1). This system is subjected to a pressure point source placed at $\left(x_{s}, y_{s} z_{s}\right)$,

$$
\hat{f}(x, y, z, t)=\delta\left(x-x_{s}\right) \delta\left(y-y_{s}\right) \delta\left(z-z_{s}\right) \mathrm{e}^{\mathrm{i} \omega t}
$$

where $\delta\left(x-x_{s}\right), \delta\left(y-y_{s}\right)$ and $\delta\left(z-z_{s}\right)$ are Dirac-delta functions, and $\omega$ is the frequency of the source.

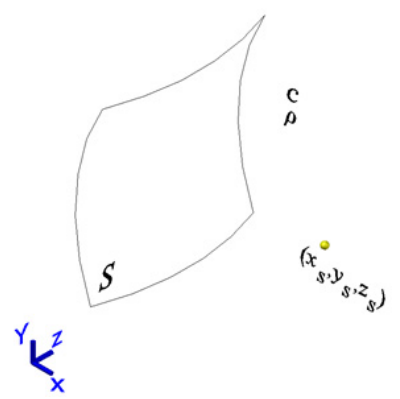

Figure 1: The geometry of the problem.

\subsubsection{Incident pressure field}

The incident pressure field generated by this source can be expressed as

$$
p_{\text {inc }}\left(x, y, z, x_{s}, y_{s}, z_{s}, \omega\right)=\frac{\mathrm{e}^{\mathrm{i} k_{c}\left(c t-r_{0}\right)}}{r_{0}},
$$

in which $k_{c}=\frac{\omega}{c}, \mathrm{i}=\sqrt{-1}$ and $r_{0}=\sqrt{\left(x-x_{s}\right)^{2}+\left(y-y_{s}\right)^{2}+\left(z-z_{s}\right)^{2}}$.

The pressure $(p)$ at any point of the spatial 3D acoustic domain can be computed by using the Helmholtz equation:

$$
\left(\frac{\partial^{2}}{\partial x^{2}}+\frac{\partial^{2}}{\partial y^{2}}+\frac{\partial^{2}}{\partial z^{2}}\right) p(\boldsymbol{x}, \omega)+\left(k_{c}\right)^{2} p(\boldsymbol{x}, \omega)=0,
$$

in which $\boldsymbol{x}=(x, y, z)$. 


\subsubsection{Normal derivative integral equation}

The normal derivative integral equation can be derived by applying the gradient operator to the boundary integral equation,

$$
b p\left(\boldsymbol{x}_{0}, \omega\right)=-\int_{S} H\left(\boldsymbol{x}, \mathbf{n}_{n 1}, \boldsymbol{x}_{0}, \omega\right) p(\boldsymbol{x}, \omega) \mathrm{d} s+p_{\text {inc }}\left(\boldsymbol{x}_{0}, \boldsymbol{x}_{s}, \omega\right)
$$

where $H$ represent the Green's functions for pressure gradient $(q)$ at a point $\boldsymbol{x}$ on the boundary $S$ due to a virtual point pressure source at a collocation point $\boldsymbol{x}_{0}=\left(x_{0}, y_{0}, z_{0}\right) . \mathbf{n}_{n 1}$ represents the unit outward normal along the boundary $S$, at $\boldsymbol{x}$. The factor $b$ takes the value $1 / 2$ if $\boldsymbol{x}_{0} \in S$ and 1 otherwise.

The Green's functions for pressure gradients in an unbounded medium, in Cartesian coordinates, can be given by:

$$
H\left(\boldsymbol{x}, \mathbf{n}_{n 1}, \boldsymbol{x}_{0}, \omega\right)=\frac{\mathrm{e}^{-\mathrm{i} k_{c} r}\left(-\mathrm{i} k_{c} r-1\right)}{4 \pi r^{2}} \frac{\partial r}{\partial \mathbf{n}_{n 1}},
$$

with $r=\sqrt{\left(x-x_{0}\right)^{2}+\left(y-y_{0}\right)^{2}+\left(z-z_{0}\right)^{2}}$.

The application of the gradient operator to eqn (4), which can be seen as assuming the existence of dipole pressure sources (dynamic doublets), leads to

$$
\text { a } p\left(\boldsymbol{x}_{0}, \omega\right)=-\int_{S} \bar{H}\left(\boldsymbol{x}, \mathbf{n}_{n 1}, \mathbf{n}_{n 2}, \boldsymbol{x}_{0}, \omega\right) p(\boldsymbol{x}, \omega) \mathrm{ds}+\bar{p}_{i n c}\left(\boldsymbol{x}_{0}, \mathbf{n}_{n 2}, \boldsymbol{x}_{s}, \omega\right)
$$

The Green's functions $\bar{H}$ are defined by applying the traction operator to $H$, which can be seen as the derivatives of these former Green's functions, to obtain pressure gradients. In these equations, $\mathbf{n}_{n 2}$ is the unit outward normal to the boundary $S$ at the collocation points $\boldsymbol{x}_{0}$, defined by the vector $\mathbf{n}_{n 2}$. In this equation, the factor $a$ is null for piecewise planar boundary elements.

The required three-dimensional Green's functions for an unbounded space are now defined as:

$$
\bar{H}\left(\boldsymbol{x}, \mathbf{n}_{n 1}, \mathbf{n}_{n 2}, \boldsymbol{x}_{0}, \omega\right)=\frac{\partial H}{\partial x} \frac{\partial x}{\partial \mathbf{n}_{n 2}}+\frac{\partial H}{\partial y} \frac{\partial y}{\partial \mathbf{n}_{n 2}}+\frac{\partial H}{\partial z} \frac{\partial z}{\partial \mathbf{n}_{n 2}}
$$

$$
\begin{gathered}
\text { with } \frac{\partial H}{\partial x}\left(\boldsymbol{x}, \mathbf{n}_{n 1}, \mathbf{n}_{n 2}, \boldsymbol{x}_{0}, \omega\right)=\frac{1}{4 \pi}\left\{A\left[\left(\frac{\partial r}{\partial x}\right)^{2} \frac{\partial x}{\partial \mathbf{n}_{n 1}}+\frac{\partial r}{\partial x} \frac{\partial r}{\partial y} \frac{\partial y}{\partial \mathbf{n}_{n 1}}+\frac{\partial r}{\partial x} \frac{\partial r}{\partial z} \frac{\partial z}{\partial \mathbf{n}_{n 1}}\right]+B\left[\frac{\partial x}{\partial \mathbf{n}_{n 1}}\right]\right\}, \\
\frac{\partial H}{\partial y}\left(\boldsymbol{x}, \mathbf{n}_{n 1}, \mathbf{n}_{n 2}, \boldsymbol{x}_{0}, \omega\right)=\frac{1}{4 \pi}\left\{A\left[\frac{\partial r}{\partial x} \frac{\partial r}{\partial y} \frac{\partial x}{\partial \mathbf{n}_{n 1}}+\left(\frac{\partial r}{\partial y}\right)^{2} \frac{\partial y}{\partial \mathbf{n}_{n 1}}+\frac{\partial r}{\partial y} \frac{\partial r}{\partial z} \frac{\partial z}{\partial \mathbf{n}_{n 1}}\right]+B\left[\frac{\partial y}{\partial \mathbf{n}_{n 1}}\right]\right\}, \\
\frac{\partial H}{\partial z}\left(\boldsymbol{x}, \mathbf{n}_{n 1}, \mathbf{n}_{n 2}, \boldsymbol{x}_{0}, \omega\right)=\frac{1}{4 \pi}\left\{A\left[\frac{\partial r}{\partial x} \frac{\partial r}{\partial z} \frac{\partial x}{\partial \mathbf{n}_{n 1}}+\frac{\partial r}{\partial y} \frac{\partial r}{\partial z} \frac{\partial y}{\partial \mathbf{n}_{n 1}}+\left(\frac{\partial r}{\partial z}\right)^{2} \frac{\partial z}{\partial \mathbf{n}_{n 1}}\right]+B\left[\frac{\partial z}{\partial \mathbf{n}_{n 1}}\right]\right\}, \\
A=-\frac{k_{c}^{2} \mathrm{e}^{-i k_{c} r}}{r}+\frac{3 i k_{c} e^{-\mathrm{e}_{c} r}}{r^{2}}+\frac{3 \mathrm{e}^{-i k_{c} r}}{r^{3}} \text { and } B=-\frac{\mathrm{i} k_{c} \mathrm{e}^{-i k_{c} r}}{r^{2}}-\frac{\mathrm{e}^{-i k_{c} r}}{r^{3}}
\end{gathered}
$$


In equation (6) the incident field is computed as

$$
\bar{p}_{i n c}\left(\boldsymbol{x}, \mathbf{n}_{n 2}, \boldsymbol{x}_{s}, \omega\right)=\frac{\mathrm{e}^{-i k_{c} r_{0}}\left(-\mathrm{i} k_{c} r_{0}-1\right)}{r_{0}^{2}}\left(\frac{\partial r_{0}}{\partial x} \frac{\partial x}{\partial \mathbf{n}_{n 2}}+\frac{\partial r_{0}}{\partial y} \frac{\partial y}{\partial \mathbf{n}_{n 2}}+\frac{\partial r_{0}}{\partial z} \frac{\partial z}{\partial \mathbf{n}_{n 2}}\right),
$$

The global solution is found by solving equation (6). This requires the discretization of the interface $S$, the boundary of the obstacle. In this analysis the interface is discretized with $N$ planar boundary elements, with one nodal point in the centre of each element.

The required integrations in these equations are evaluated using a Gaussian quadrature scheme when they are not performed along the loaded element. For the loaded element, the existing hypersingular integrands of the Green's functions are calculated analytically.

\subsubsection{Analytical Integration of the hypersingular integral}

Consider the singular rectangular element of width $\mathrm{W}$ (in the $x$ direction) and length $\mathrm{L}$ (in the $z$ direction) shown in Figure 2:

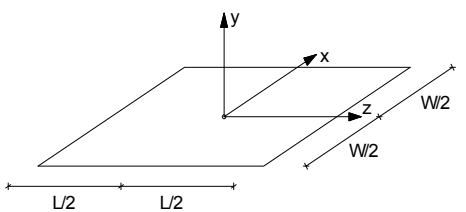

Figure 2: Geometry of the boundary element.

The integration of the Green's function $\int_{-L / 2}^{L / 2} \int_{-W / 2}^{W / 2} \bar{H}\left(\boldsymbol{x}, \mathbf{n}_{n 1}, \mathbf{n}_{n 2}, \boldsymbol{x}_{0}, \omega\right) d x d z$ (with $\mathbf{n}_{n 2}=\mathbf{n}_{n 1}$ ) leads to a hypersingular term.

The evaluation of this integration is performed by writing $\bar{H}\left(\boldsymbol{x}, \mathbf{n}_{n 1}, \mathbf{n}_{n 2}, \boldsymbol{x}_{0}, \omega\right)$ as the sum of two-dimensional Green's functions with varying spatial wavenumbers. This is accomplished by first applying a Fourier transformation in the $z$ direction to the three-dimensional Green's function

$$
G\left(\boldsymbol{x}, \boldsymbol{x}_{0}, \omega\right)=\frac{\mathrm{e}^{-\mathrm{i} k_{c} r}}{4 \pi r},
$$

The application of a spatial Fourier transformation to this in that direction leads to a line pressure field, whose amplitude varies sinusoidally in the third dimension $(z)$,

$$
\widehat{G}\left(x, y, x_{0}, y_{0}, k_{z}, \omega\right)=\frac{-\mathrm{i}}{8 \pi} H_{0}\left(k \bar{r}_{0}\right) \mathrm{e}^{-\mathrm{i} k_{z} z},
$$


in which $H_{n}(\ldots)$ are second kind Hankel functions of the order $n, k=\sqrt{\frac{\omega^{2}}{c^{2}}-k_{z}^{2}}$, with $\operatorname{Im}(k)<0, \bar{r}_{0}=\sqrt{\left(x-x_{0}\right)^{2}+\left(y-y_{0}\right)^{2}}$, where $k_{z}$ is the wavenumber in the $z$ direction.

Assuming the presence of an infinite set of equally-spaced virtual sources in the $z$ direction, the former Green's function (in eqn. 9) can be written as:

$$
G\left(\boldsymbol{x}, \boldsymbol{x}_{0}, \omega\right)=\frac{2 \pi}{L_{v s}} \sum_{m=-\infty}^{\infty} \hat{G}\left(x, y, x_{0}, y_{0}, k_{z}, \omega\right) \mathrm{e}^{-\mathrm{i} k_{z m} z},
$$

where $L_{v s}$ is the spatial source interval, and $k_{z m}=\frac{2 \pi}{L_{v s}} m$.

This equation converges and can be approximated by a finite sum of terms (M). The distance $L_{v s}$ needs to be large enough to avoid spatial contamination. The use of complex frequencies further reduces the influence of the neighbouring fictitious sources. The 3D Green's field can therefore be computed as the pressure irradiated by a sum of harmonic (steady-state) line loads, whose amplitude varies sinusoidally in the $z$ dimension.

Using the procedure above, the evaluation of the integration $\int_{-L / 2}^{L / 2} \int_{-W / 2}^{W / 2} \bar{H}\left(\boldsymbol{x}, \mathbf{n}_{n 1}, \mathbf{n}_{n 2}, \boldsymbol{x}_{0}, \omega\right) d x d z$ can be performed by writing $\bar{H}\left(\boldsymbol{x}, \mathbf{n}_{n 1}, \mathbf{n}_{n 2}, \boldsymbol{x}_{0}, \omega\right)$ as the sum of two-dimensional Green's functions with varying spatial wavenumbers. This can be accomplished by applying the traction operator to the Green's function

$$
\hat{H}\left(x, y, \mathbf{n}_{n 1}, x_{0}, y_{0}, k_{z}, \omega\right)=\frac{\mathrm{i} k}{8 \pi} H_{1}\left(k \bar{r}_{0}\right)\left(\frac{\partial \bar{r}_{0}}{\partial x} \frac{\partial x}{\partial \boldsymbol{n}_{n 1}}+\frac{\partial \bar{r}_{0}}{\partial y} \frac{\partial y}{\partial \boldsymbol{n}_{n 1}}\right) \mathrm{e}^{-\mathrm{i} k_{z} z},
$$

which leads to

$$
\bar{H}\left(x, y, \mathbf{n}_{n 1}, \mathbf{n}_{n 1}, x_{0}, y_{0}, k_{z}, \omega\right)=\frac{\mathrm{i} k}{8 \pi}\left[\begin{array}{l}
-k H_{2}\left(k \overline{r_{0}}\right)\left(\frac{\partial \bar{r}_{0}}{\partial x} \frac{\partial x}{\partial \mathbf{n}_{n l}}+\frac{\partial \bar{r}_{0}}{\partial y} \frac{\partial y}{\partial \mathbf{n}_{n l}}\right)^{2}+ \\
\frac{H_{1}\left(k \bar{r}_{0}\right)}{\bar{r}_{0}}
\end{array}\right] \mathrm{e}^{-\mathrm{i} k_{z} z},
$$

This procedure allows the integration of $\int_{-L / 2}^{L / 2} \int_{-W / 2}^{W / 2} \bar{H}\left(\boldsymbol{x}, \mathbf{n}_{n 1}, \mathbf{n}_{n 2}, \boldsymbol{x}_{0}, \omega\right)$ to be obtained as

$$
\int_{-L / 2}^{L / 2} \int_{-W / 2}^{W / 2} \bar{H}\left(\boldsymbol{x}, \mathbf{n}_{n 1}, \mathbf{n}_{n 2}, \boldsymbol{x}_{0}, \omega\right) d x d z=\frac{\mathrm{i}}{2 L_{v s}} \sum_{m=-M}^{M} \int_{-L / 2}^{L / 2} I_{2}\left(k \bar{r}_{0}\right) \mathrm{e}^{-\mathrm{i} k_{z m} z} d z,
$$

with

$$
\begin{aligned}
& \int_{-L / 2}^{L / 2} I_{2}\left(k \bar{r}_{0}\right) \mathrm{e}^{-\mathrm{i} k_{z m} z} d z=I_{2}\left(k \overline{r_{0}}\right) L \quad \text { if } \quad m=0 \\
& \int_{-L / 2}^{L / 2} I_{2}\left(k \bar{r}_{0}\right) \mathrm{e}^{-\mathrm{i} k_{z m} z} d z=I_{2}\left(k \bar{r}_{0}\right) \frac{2 \sin \left(k_{z m} \frac{L}{2}\right)}{k_{z m}} \quad \text { if } \quad m \neq 0
\end{aligned}
$$


where $I_{2}\left(k \bar{r}_{0}\right)=k^{2}\left[\int_{0}^{W / 2} H_{0}\left(k \overline{r_{0}}\right) d r-\frac{1}{k} H_{1}\left(k \frac{W}{2}\right)\right]$ with

$\int_{0}^{W / 2} H_{0}\left(k \overline{r_{0}}\right) d r=\frac{W}{2} H_{0}\left(k \frac{W}{2}\right)+\pi \frac{L}{4}\left[H_{1}\left(k \frac{W}{2}\right) S_{0}\left(k \frac{W}{2}\right)-H_{0}\left(k \frac{W}{2}\right) S_{1}\left(k \frac{W}{2}\right)\right]$,

where $S_{n s}(\ldots)$ are Struve functions of order $n s$.

\subsection{Corner-space domain}

To avoid having to discretize the horizontal and vertical interface which simulates the floor and building façade, the above Green's functions and the incident pressure need to be rewritten in a way that satisfies null normal velocities at this boundary (assumed to be rigid). This can be accomplished by adding the pressure field generated by the real source to that produced by virtual sources (image sources), which are located in such a way that they act as mirrors of the real source in relation to the vertical and horizontal planes. The Green's functions are not presented here since this is a straightforward procedure.

\section{Numerical applications}

Figure 3 illustrates the two geometries simulated to evaluate the influence of the longitudinal barrier shape of a 3D barrier on the sound attenuation on a building façade. They are: Case I - barrier with a horizontal sinusoidal variation with a wavelength of $6.0 \mathrm{~m}$; Case II - barrier with a horizontal sinusoidal variation with a wavelength of $1.5 \mathrm{~m}$. The amplitude of the sinusoidal variation is kept constant at $0.25 \mathrm{~m}$. All barriers are thin, $3.0 \mathrm{~m}$ high, $6.0 \mathrm{~m}$ long and placed parallel to a tall building, with their axes $5.0 \mathrm{~m}$ from the building façade. The host acoustic medium is air.

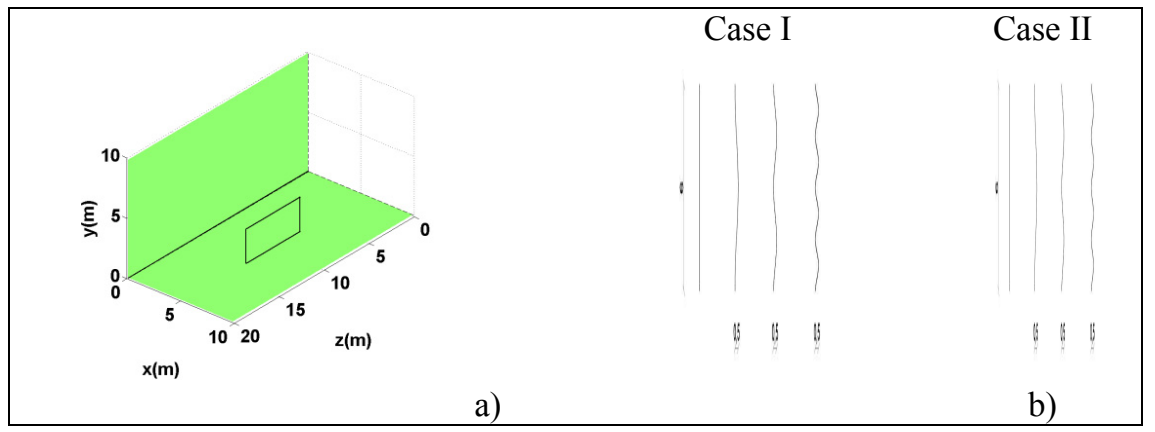

Figure 3: Geometry used on the numerical applications: a) 3D view; b) horizontal cross-sections of the geometries used in the numerical applications: Case I - barrier with a horizontal sinusoidal variation with a wavelength of $6.0 \mathrm{~m}$; Case II - barrier with a horizontal sinusoidal variation with a wavelength of $1.5 \mathrm{~m}$. 
The 3D source is placed at $(7 \mathrm{~m}, 2 \mathrm{~m}, 10 \mathrm{~m})$. Pressure computations are performed in the frequency range of $[4.25,1088.0 \mathrm{~Hz}]$ with a frequency step of $4.25 \mathrm{~Hz}$ over fine grids of receivers placed over four planes: $x=0.1 \mathrm{~m}$, $y=0.1 \mathrm{~m}, z=0.0 \mathrm{~m}$ and $z=10.0 \mathrm{~m}$. The receivers were spaced at equal intervals of $0.2 \mathrm{~m}$ in the $\mathrm{x}, \mathrm{y}$ and $\mathrm{z}$ directions.

Time responses were then obtained by applying an inverse Fourier transformation with the source temporal variation reproducing a Ricker pulse with a characteristic frequency of $400.0 \mathrm{~Hz}$.

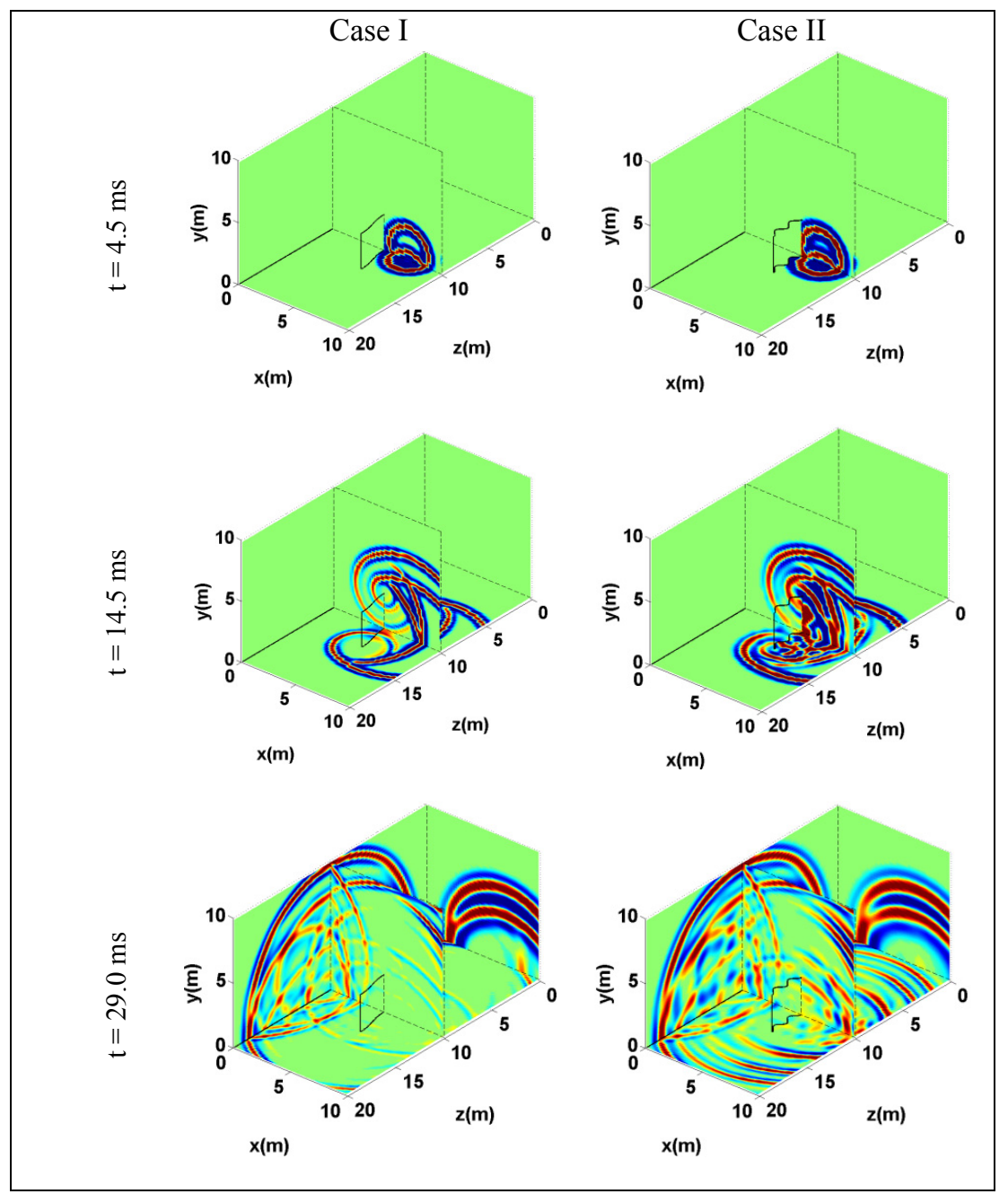

Figure 4: Pressure responses at time instants $t=4.5 \mathrm{~ms}, t=14.5 \mathrm{~ms}$ and $t=29.0 \mathrm{~ms}$. 
Figure 4 contains the time domain snapshots showing the pressure wavefield at different time instants ( $t=4.5 \mathrm{~ms}, t=14.5 \mathrm{~ms}$ and $t=29.0 \mathrm{~ms}$ ). At each instant three plots are displayed for each of the 4 cases.

At $t=4.5 \mathrm{~ms}$, Figure 4 shows, for all cases, that part of the incident pulse has been reflected by the ground and other part reaches the barrier. The result of the diffraction of these two pulses by the top of the barrier is clearly seen at $t=14.5 \mathrm{~ms}$. The reflections of the previous pulses at the barrier are also quite visible, travelling backwards. In addition, the diffraction caused by the lateral wedges of the barrier has generated a set of pulses that are present for all cases. It can be seen that the more undulating barriers (with a smaller horizontal wavelength variation) generate a more complex diffracted field, particularly visible on the lateral barrier wedges, given the reflections from the curved surface of the barrier.

Pulses that were previously diffracted at the top and at the lateral wedges of the barrier have already reached the ground behind the barrier and the building façade whence they were reflected back at $t=29.0 \mathrm{~ms}$, creating a complex pattern of pulses. Among the different pressure fields created by the different barriers, it can be seen that the more undulating barriers exhibit more complex wavefields. It can further be observed that in the presence of those barriers pressures pulses of more enhanced amplitude travel in the lateral directions after being reflected from the barriers' surface.

\subsection{Sound pressure level attenuation}

From the time response it is not easy to conclude if the longitudinal shape influences the sound attenuation provided by a $3 \mathrm{D}$ rigid acoustic barrier on a building façade, therefore the sound pressure level attenuation provided by the different barriers is computed in this section.

The time domain responses are the basis for calculating the sound pressure level SPL) over the same grid of receivers used before. The expression $10 \log \left(p^{2} /\left(2 \times 10^{-5}\right)^{2}\right)$ is used to calculate the SPL in $\mathrm{dB}$, where $p$ refers to the maximum amplitude of the time responses. Thus the later pulses arriving at the grid of receivers, produced by the multiple reverberations between the building façade and the barrier, do not contribute to the final value for the SPL because their amplitude decays. The SPL attenuation is calculated as the difference between the SPL obtained when there is no acoustic barrier and the SPL calculated in the presence of an acoustic barrier.

Figure 5 displays the sound pressure level (the left plots), calculated as described above, and its attenuation (the right plots) when there is a barrier between the source and the building. 


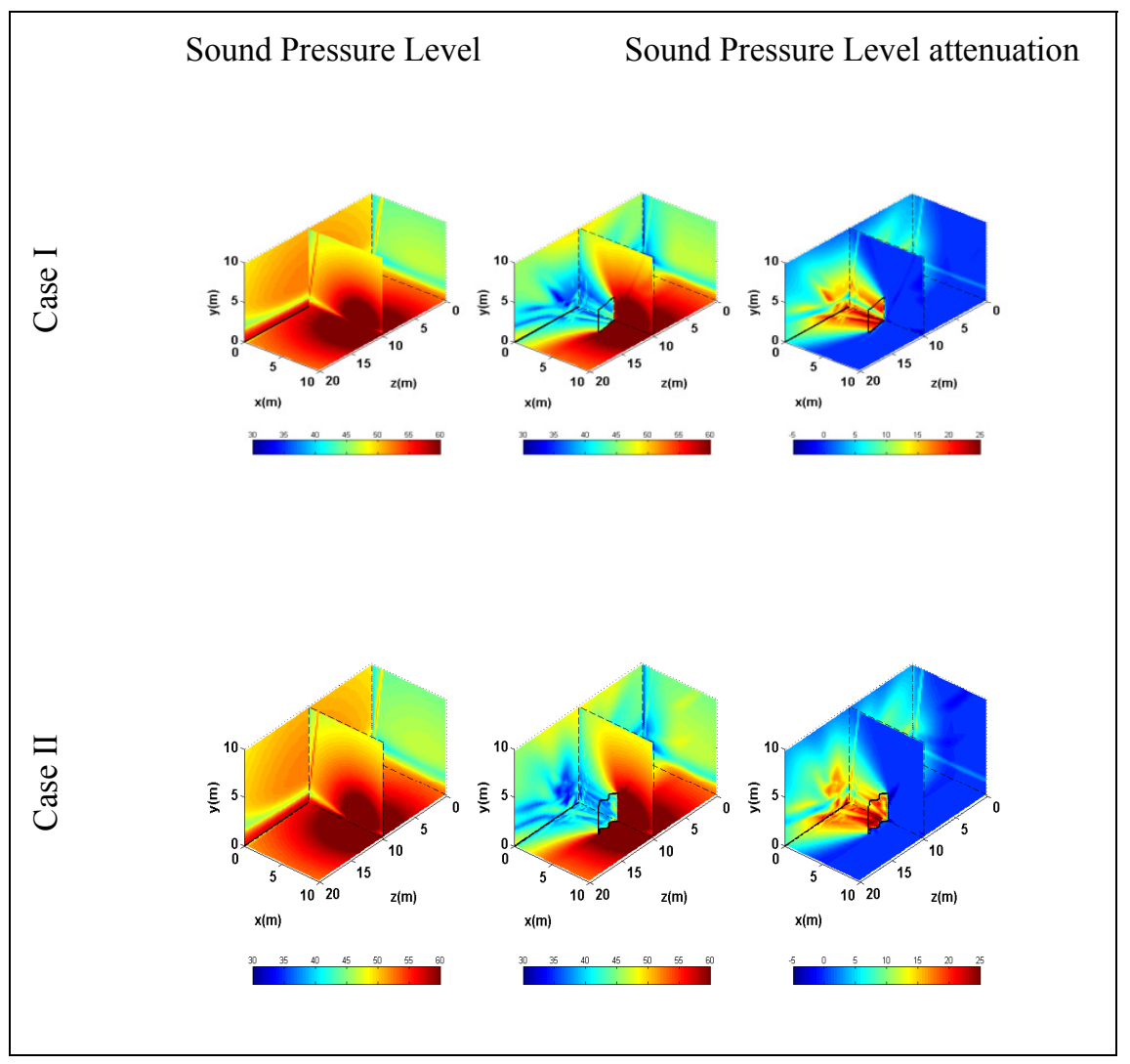

Figure 5: $\quad$ Sound pressure level and attenuation at the grids of receivers over four planes: $x=0.1 \mathrm{~m}, y=0.1 \mathrm{~m}, z=0.0 \mathrm{~m}$ and $z=10.0 \mathrm{~m}$.

The results obtained indicate an SPL attenuation of $15-25 \mathrm{~dB}$ behind the barriers. The more undulating barriers generate more pronounced SPL attenuation, as recorded at receivers placed further away from the central plane $z=10.0 \mathrm{~m}$. Negative SPL attenuation is registered in front of the barrier, given that the SPL provided by the barrier is greater because the reflections of the sound pulses on its surface. A general reduction in the sound pressure level is noted in front of the barrier for the less undulating barriers. On the building façade the most undulating barrier performs a little better, particularly for receivers in the vicinity of the central plane. A general reduction in the sound pressure level attenuation is noted with increasing distance between the receiver and the source.

The sound pressure level and its attenuation were also calculated for pulses with different frequency characteristics. The results revealed similar features (not illustrated). 


\section{Conclusions}

A boundary element method based on the normal derivative integral equation to model acoustic wave propagation in the vicinity of three-dimensional thin acoustic elements when the medium is perturbed by fixed 3D sources has been proposed in this paper. The solution was defined in the frequency domain. The integrals' integrations are evaluated using a Gaussian quadrature scheme when they are not performed for the singular element. The hypersingular integrals that appear when the element to be integrated is the loaded one (singular element) are integrated analytically. The proposed algorithm was verified against analytical solutions derived for cylindrical circular geometries and showed a high degree of accuracy.

Numerical applications were used to evaluate the effect of the longitudinal geometry of 3D thin acoustic barriers on the sound pressure level attenuation on a building façade. The analysis of time domain snapshots shows consistency with the physics of the problem and made it possible to verify a more complex pressure wavefield for the more undulating barriers. The sound pressure level and the attenuation provided by the acoustic barrier reflected the phenomena registered in the time responses. Receivers in the immediate vicinity of the ground show that the interaction between the direct field, diffracted by the edge of the barrier, and that first reflected from the ground leads to a poorer performance by the barrier. The use of undulating barriers ensures a slight increase in the sound pressure level attenuation, particularly for receivers placed on the building façade, behind the barrier.

\section{References}

[1] Marburg, S., Nolte, B., Computational Acoustics of Noise Propagation in Fluids: Finite and Boundary Element Methods, Springer-Verlag: Berlin, 2008.

[2] Cheng, A.H.D., Cheng D.T., Heritage and early history of the boundary element method. Engineering Analysis with Boundary Elements, 29, pp. 268-302, 2005.

[3] Pluymers, B., Van Hal B., Vandepitte D., Desmet W., Trefftz-Based Methods for Time-Harmonic Acoustics. Archives of Computational Methods in Engineering, 14, pp. 343-381, 2007.

[4] Jensen F.B., Kuperman W.A., Porter M.B., Schmidt H., Computational Ocean Acoustics, Springer-Verlag: New York, 2000.

[5] Grilli, S., Pedersen, T., Stepanishen P., A Hybrid Boundary Element Method for Shallow Water Acoustic Propagation over an Irregular Bottom. Engineering Analysis with Boundary Elements, 21, pp. 131-145, 1998.

[6] Santiago, J.A.F., Wrobel L.C., A Boundary Element Model for Underwater Acoustics in Shallow Water. Computer Modeling in Engineering \& Sciences, 1, pp. 73-80, 2000. 
[7] Pereira, A., Tadeu, A., Godinho, L., Santiago, J.A.F., 2.5D BEM modeling of underwater sound scattering in the presence of a slippage interface separating two flat layered regions. Wave Motion, 47, pp. 676-692, 2010.

[8] Portela, A., Aliabadi, M.H., Rooke, D.P., The dual boundary element method: effective implementation for crack problems. International Journal for Numerical Methods in Engineering, 33, pp. 1269-1287, 1992.

[9] Chen, J.T., Hong, H.K., Review of dual boundary element methods with emphasis on hypersingular integrals and divergent series. Applied Mechanics Reviews, 52, pp. 17-33, 1999.

[10] Krishnasamy, G., Rizzo, F.J., Lie, Y., Boundary integral-equations for thin bodies. International Journal for Numerical Methods in Engineering, 37, pp. 107-121, 1994.

[11] Amado Mendes, P., Tadeu, A., Wave propagation in the presence of empty cracks in an elastic medium. Computational Mechanics, 38, pp. 183-199, 2006.

[12] Lacerda, L.A., Wrobel, L.C., Mansur, W.J., A dual boundary element formulation for sound propagation around barriers over an impedance plane. Journal of Sound and Vibration, 202, pp. 235-247, 1997.

[13] Lacerda, L.A., Wrobel, L.C., Power, H., Mansur, W.J., A novel boundary integral formulation for three-dimensional analysis of thin acoustic barriers over an impedance plane. Journal of the Acoustical Society of America, 104, pp. 671-678, 1998.

[14] Antonio, J., Tadeu, A., Amado Mendes, P., A $2.5 D$ traction boundary element method formulation applied to the study of wave propagation in a fluid layer hosting a thin rigid body. Journal of Computational Acoustics, 16, pp. 177-198, 2008. 